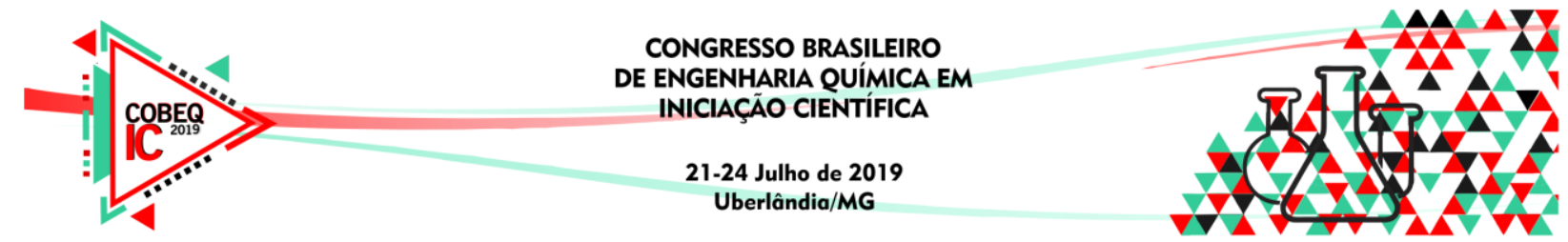

\title{
ESTUDO DE METABÓLITOS SECUNDÁRIOS E INCORPORAÇÃO DO EXTRATO GLICERINADO DA Pyrostegia venusta EM LOÇÃO HIDRATANTE
}

\author{
P. S. MAGALHÃES ${ }^{1}$, D.C. S. FREITAS ${ }^{1}$,J. L. C. $\operatorname{COSTA}^{1}$ e M. P. O. RAMOS${ }^{2}$. \\ ${ }^{1}$ Graduando em Engenharia Química - Centro Universitário de Patos de Minas - UNIPAM. \\ ${ }^{2}$ Professor do curso de Engenharia Química - Centro Universitário de Patos de Minas - \\ UNIPAM. \\ E-mail para contato: patriciasilva@unipam.edu.br
}

\begin{abstract}
RESUMO - O uso de plantas para fins medicinais, nutricionais e estéticos, remota a antiguidade e esta inserido em todas as culturas. Algumas partes da Pyrostegia venusta, uma planta do cerrado, conhecida como Cipó-de-São-João tem aplicações na medicina popular, e o extrato alcoólico de suas folhas e flores tem sido utilizados no tratamento do vitiligo. Com isso, o presente trabalho tem como objetivo identificar os metabolitos secundários e a atividade antioxidante da $P$.venusta para posteriormente incorporar o extrato glicerinado de sua raiz em uma loção hidratante. $\mathrm{Na}$ identificação dos metabolitos foram utilizados testes de mudança de cor e/ou precipitação e para a atividade antioxidante foi utilizado o método de captura de radicais livres DPPH com adaptações. Adicionalmente foram realizados testes físico-químicos na loção para avaliar a estabilidade da mesma. Foi identificado a presença de flavonoides nas raízes da Pyrostegia venusta, onde apresentou para a atividade antioxidante um valor alto de $\mathrm{IC}_{50}$ sendo de $156,4 \mu \mathrm{g} / \mathrm{mL}$ e os resultados físico-químicos ficaram dentro dos parâmetros da ANVISA.
\end{abstract}

\section{INTRODUÇÃO}

A Pyrostegia venusta, conhecida popularmente como Cipó-de-São-João, pertence à família das Bignoniceae, é uma trepadeira lenhosa, distribuída por todo o Brasil, exceto no Norte. Ela pode ser encontrada nos declives das matas, em campos abertos, na beira das estradas e em pastos, onde já foi registrado morte de bovinos por envenenamento. Algumas partes dessa planta têm aplicação na medicina popular, a qual também é bastante utilizada em ornamentações (Ferreira et al., 2000).

Os princípios ativos utilizados na farmacologia são extraídos dos metabólitos secundários, que se dispõem em baixa quantidade na maioria das famílias das plantas, substâncias essas, que estão diretamente ligadas à adaptação do vegetal ao meio ambiente em que vive (Correia et al., 2006).

Com estudos recentes apontados por Silva et al. (2011), os extratos alcoólicos das flores e folhas do Cipó-de-São-João tem sido utilizados para o tratamento de vitiligo.O extrato 


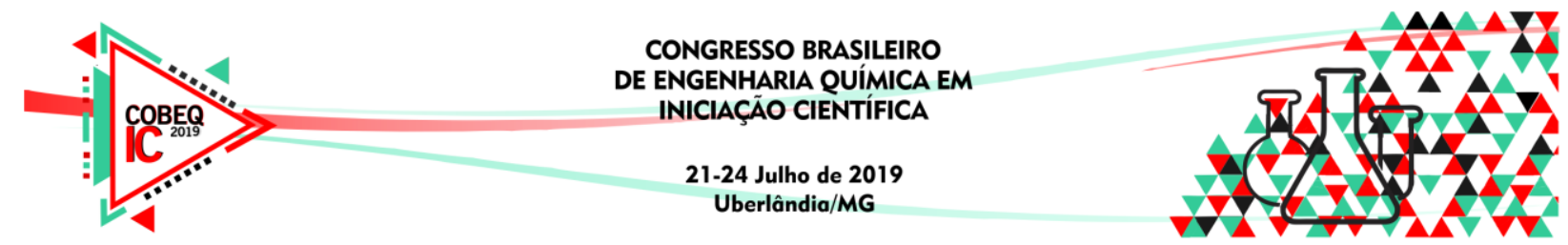

hidroetanólico das flores apresentam potencialidades biológicas na melhora dos sintomas da gripe e o extrato etanólico causa a baixa na genotoxidade no teste de aberrações cromossômicas e micronucleares, feitos em ratos.

O presente trabalho tem como objetivo realizar análise de prospecção química para identificar metabolitos especiais, a fim de produzir uma loção hidratante com parâmetros físico-quimicos a partir do extrato da Pyrostegia venusta, visando amenizar um dos sintomas do vitiligo.

\section{METODOLOGIA}

As raízes da planta foram colhidas pela manhã fora da estação de floração, após o trevo da Av. Marabá com a Rod. Sebastião Alves do Nascimento (latitude -18,5753 e longitude 46,4436) em Patos de Minas (MG).

O material foi seco em estufa ICAMC a $60^{\circ} \mathrm{C}$, e depois levado a um moinho de facas. A identificação de flavonóides, a análise de DPPH e os testes físico-químicos foram feitos no Laboratório de Química Orgânica do Centro Universitário de Patos de Minas.

\subsection{Identificação de Flavonóides na Raiz da P.venusta}

Para identificação de flavonóides foram realizados quatro testes com extrato etanólico, sendo eles o teste de Shinoda que utiliza fitas de magnésio e ácido clorídrico; o teste NP-PEG por cromatografia com solução metanólica de difeniboriloxietilamina a $2 \%$ mais solução etanólica de polietilenoglicol a $5 \%$, observada sob luz ultravioleta $(=365 \mathrm{~nm})$; o teste de Cloreto de alumínio comparado sob luz ultravioleta; e o teste de Pew que utiliza metanol, zinco metálico e acido clorídrico concentrado (Santos et. al, 2018).

\subsection{Avaliação da Atividade Antioxidante das Raízes da Pyrostegia venusta}

Os flavonóides identificados na raiz da Pyrostegia venusta possibilitaram a análise de atividades antioxidante através do método de captura de radicais livres DPPH (2,2-difenil-1picril-hidrazil).

O extrato foi obtido por percolação por $24 \mathrm{~h}$, usando etanol PA como solvente extrator. Após filtração e secagem, uma solução estoque foi obtida usando $0,024 \mathrm{~g}$ do extrato diluído em $50 \mathrm{~mL}$ de álcool etílico, retirando alíquotas de $0,1 \mathrm{~mL}, 0,2 \mathrm{~mL}, 0,4 \mathrm{~mL}, 0,6 \mathrm{~mL}, 0,8 \mathrm{~mL}$ e $1,0 \mathrm{~mL}$ com o auxílio de um pipetador automático, adicionando em cada $2 \mathrm{~mL}$ de DPPH em concentração de $0,004 \%(\mathrm{~m} / \mathrm{V})$ e aferiu-se com etanol até $10 \mathrm{~mL}$ nas seis soluções.

Assim obtiveram-se concentrações do extrato de 4,0mg/L à 40,0mg/L que foram armazenados fora do alcance de luz por 30 minutos e lidas em espectrofotômetro UV-vis em $517 \mathrm{~nm}$ para determinação da absorbância da amostra no DPPH. Como comparativo fez-se controle positivo com a solução estoque de $2 \mathrm{~g} / \mathrm{L}$ do padrão de quercetina e controle negativo com a solução DPPH em etanol. Para calcular o percentual do sequestro do radical livre foi utilizada a Equação 1. 


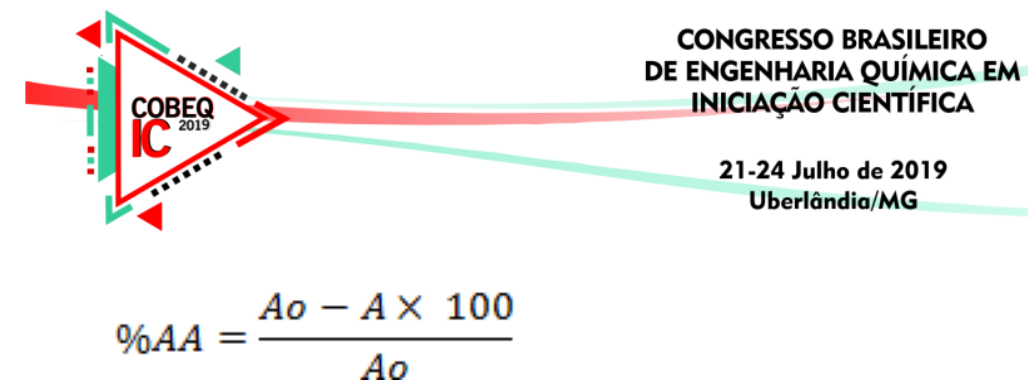

$\% \mathrm{AA}=$ percentual de atividades antioxidantes

Ao = absorbância do DPPH utilizado como controle

$\mathrm{A}=$ absorbância da amostra no DPPH

\subsection{Aplicação do Extrato Glicerinado das Raízes da P.venusta na Loção Hidratante}

O preparo do cosmético foi realizado com os componentes apresentados na Tabela 1, onde o extrato glicerinado foi extraído por uma semana, aquecido em banho-maria a $50^{\circ} \mathrm{C}$ por 20 min e filtrado.

Tabela 1 - Formulação da loção hidratante

\begin{tabular}{|c|c|c|}
\hline Componentes & Função & \% \\
\hline \hline Fase oleosa: & & \\
Polawax & Agente emulsificante & 6 \\
Vaselina liquida & Agente de consistência & 4 \\
Butilhidroxitolueno & Conservante antioxidante & 0,05 \\
\hline Fase aquosa: & & \\
Propilenoglicol & Umectante & 4 \\
Agua deionizada & Veículo & 100 \\
\hline Fase final: & & 1 \\
Silicone DC 245 & Espessante que da suavidade & 0,5 \\
Phenonip & Conservante & 0,5 \\
Essência & Aroma & 2 \\
Extratoglicerinado & Ativo com propriedades & \\
\multicolumn{2}{|c|}{ Fonte: Desconhecida. } \\
\hline
\end{tabular}

\subsection{Ensaios Físico-químicos da Loção Hidratante}

Segundo a ANVISA (2008), os ensaios físico-químicos consistem em determinar de acordo com um procedimento especifico uma ou mais características de um produto, sendo realizadas no cosmético em análise as determinações descritas na Tabela 2.

Tabela 2 - Ensaios Físico-químicos

\begin{tabular}{|c|c|}
\hline Parâmetros & Médoto Analítico \\
\hline \hline$p \mathrm{H}$ & Potenciometria \\
\hline Viscosidade & Viscosímetro \\
\hline Densidade & Densímetro \\
\hline Centrifuga & Centrifuga \\
\hline
\end{tabular}

\section{RESULTADOS E DISCUSSÕES}




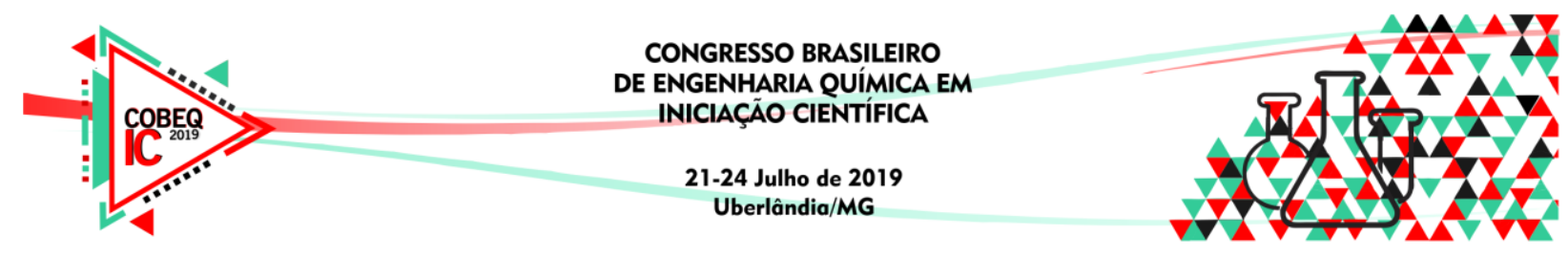

\subsection{Identificação de Flavonóides na Raiz da Pyrostegia venusta}

Através das análises de prospecção química foi comprovada a presença de flavonóides por meio dos testes de Shinoda e NP-PEG.

Os métodos de identificação dos outros metabólitos não foram realizados com a raiz, pois o cosmético produzido requer metabólitos que tenham propriedades antioxidantes podendo ser utilizadas no tratamento de amenização do vitiligo, encontrados nos flavonóides (Ribeiro, 2011).

\subsection{Atividade Antioxidante na Raiz da Pyrostegia venusta}

As análises na raiz da Pyrostegia venusta possibilitaram calcular e comparar a porcentagem de atividades antioxidante da amostra com a quercertina, pelo método de captura de radicais livres DPPH(2,2-difenil-1-picril-hidrazil) em seis concentrações diferentes, representados no Gráfico 1.

Gráfico 1 - AA\% do extrato etanólico da P.venusta em concentrações de 4 à 40 mg/L comparado com a quercetina

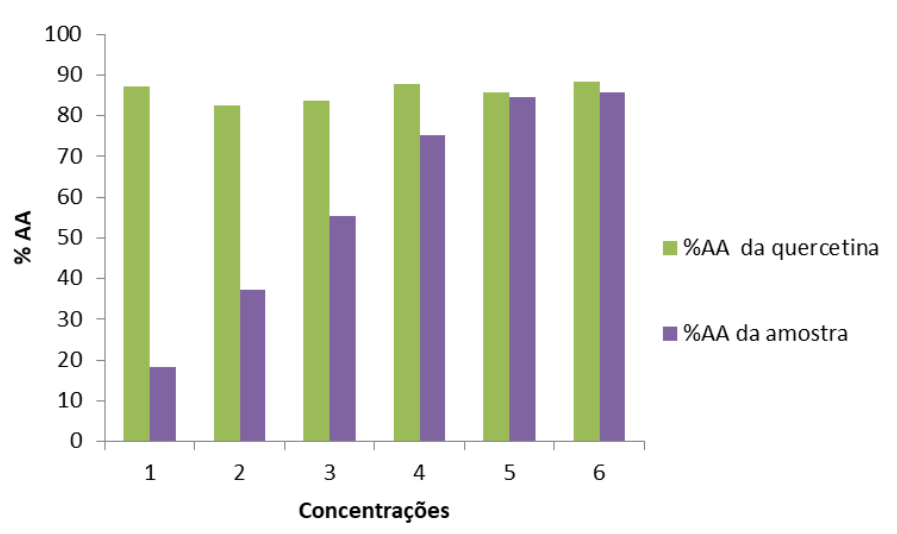

Fonte: Autores, 2018.

É possível perceber ao comparar os resultados, que a \% da atividade antioxidante do extrato etanólico da P.venusta apresenta aumento de AA\% de acordo com a concentração do extrato. Podendo promover melhora na qualidade do tecido e vitalidade da pele. Indicada para peles desnutridas, desvitalizadas, envelhecidas e atônicas.

A concentração inibidora (IC50) descreve a concentração inicial de DPPH em 50\% consumida no extrato etanoico da raiz pela atividade antioxidante, que foi calculado através da equação de regressão, onde mostra a concentração vs. \%AA do extrato.

O $\mathrm{IC}_{50}$ (quantidade necessária para atingir a inibição de $50 \%$ ) encontrado no extrato etanóico da Pyrostegia venusta apresentou valor de $156,4 \mu \mathrm{g} / \mathrm{mL}$. O valor encontrado nos 


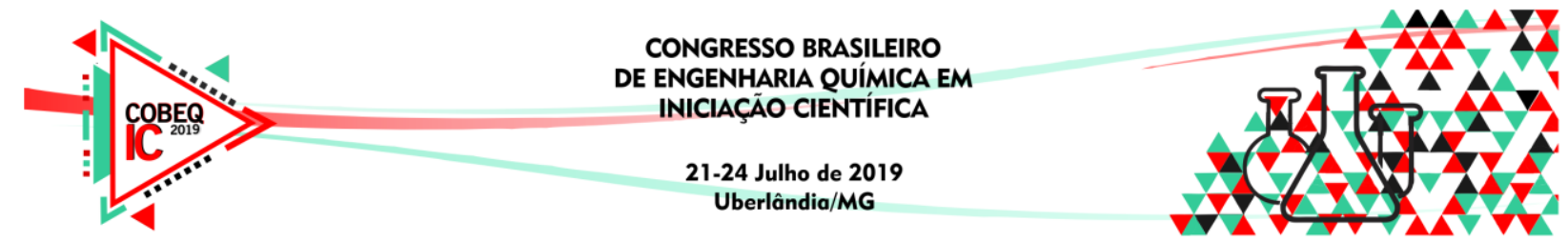

estudos de Altoé (2014) apresentou 38,62 $\mu \mathrm{g} / \mathrm{mL}$ com extrato de acetato de etila e 83,04 $\mu \mathrm{g} / \mathrm{mL}$ com extrato bruto.

\subsection{Testes Físico-químicos Realizados na Loção Hidratante}

Os resultados dos testes físico-químicos apresentados na Tabela 3 comparam os dados encontrados na loção hidratante com o extrato glicerinado da raiz da Pyrostegia venusta aos dados do estudo de Minami (2015), que desenvolveu loções à base de extratos vegetais.

Tabela 3 - Comparação dos testes físico-químicos na loção hidratante da P.venusta

\begin{tabular}{|c|c|c|}
\hline Parâmetros físico-químicos & Resultados & Minami (2015) \\
\hline \hline Viscosidade $[\mathrm{mPa} . \mathrm{s}]$ & 3.195 & 4.500 \\
\hline Densidade $\left[\mathrm{g} / \mathrm{cm}^{3}\right]$ & 0,936 & 0,877 \\
\hline$p \mathrm{H}$ & 5,11 & 4,96 \\
\hline $\begin{array}{c}\text { Centrifugação [3.500 rpm por } \\
\text { 5 minutos] }\end{array}$ & Não apresentou sobrenadante & Não apresentou sobrenadante \\
\hline
\end{tabular}

Fonte: Autores, 2018.

A pequena variação entre os resultados obtidos em estudo e aos dados na literatura pode advir do fato de que Minami (2015) usou um extrato com álcool estearílico etoxilado $21 \mathrm{e}$ álcool estearílico etoxilado 2 e/ou do fato de que sua a composição de sua loção trás os óleos das plantas Margarida (Bellis perennis), Rosa mosqueta (Rosa canina) e Centelha asiática (Centella asiatica), enquanto o presente estudo trabalha com o extrato glicerinado (álcool de cereal e glicerina) da raiz da P.venusta.

\section{CONCLUSÕES}

(i) Conclui-se que há presença de flavonoides nas raízes da Pyrostegia venusta;

(ii) Não é possível afirmar a eficácia da atividade antioxidante encontrada na raiz visto que ela é significativa para valores de IC50 menores e/ou entre $83,04 \mu \mathrm{g} / \mathrm{mL}$ e $38,62 \mu \mathrm{g} / \mathrm{mL}$, apresentando $156,4 \mu \mathrm{g} / \mathrm{mL}$ que é um valor alto de $\mathrm{IC}_{50}$.

(iii) A loção hidratante com o extrato glicerinado da raiz apresentou resultados físicoquímicos adequados de acordo com a ANVISA (2008) para a hidratação corporal e a diferença observada na comparação com Minami (2015) não é significativa o suficiente para se justificar alguma mudança na composição da loção.

(iv) São necessários estudos futuros para confirmar se a loção hidratante tem efeito em relação ao vitiligo.

\section{REFERÊNCIAS}

AGÊNCIA NACIONAL DE VIGILÂNCIA SANITÁRIA. Guia de controle de qualidade de produtos cosméticos. Brasília, 2008. 


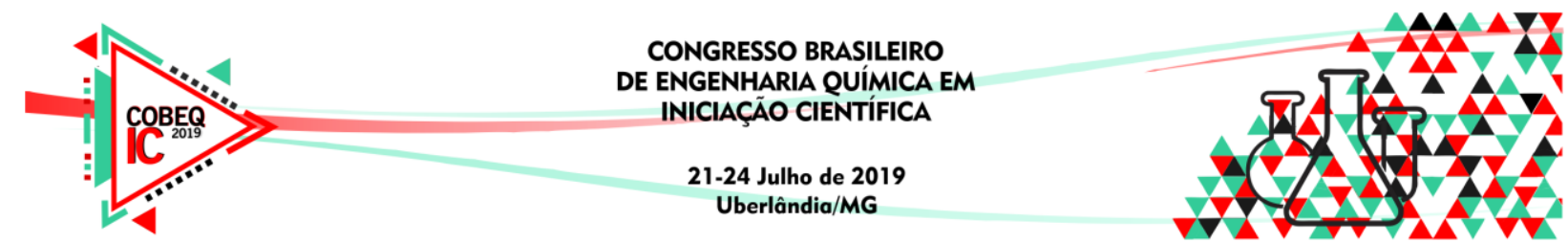

ALTOÉ, T.P. Estudo Químico-Biológico de Pyrostegia venusta (KerGawl.) Miers (Bignoniaceae). 2014. 124 f. Dissertação (Mestrado) - Curso de Química, Universidade Federal do Espírito Santo, Vitória, 2014.

CORREIA, S. J.; DAVID, J. P.; DAVID, J. M. Metabólitos secundários de espécies de Anacardiaceae. Química Nova, v. 29, n. 6, p. 1287-1300, 2006.

FERREIRA, D.T. et al. Constituintes químicos das raízes de Pyrostegia venusta e considerações sobre a sua importância medicinal. Química Nova, v. 23, n. 1, p. 42-6, 2000 .

MINAMI, S. K. et al. Desenvolvimento de loção fotoprotetora à base de extratos vegetais para a pele da mão. Interface $H S$, v. 10, n. 1, 2015.

RIBEIRO, Emmanuelle Moraes. Prospecção de flavonóides inibidores enzimáticos em espécies de Protium da Amazônia brasileira. 2011.

SANTOS, Bianca Duarte Araújo; LAHLOU, Brenda NajatBoechat; ORSOLIN, Priscila Capelari. Avaliação do potencial anticarcinogênico do extrato aquoso de romã (Punica granatum l.) por meio do teste para detecção de clones de tumores epiteliais (Warts) em Drosophila melanogaster. Saúde.com, v. 14, n. 1, 2018.

SILVA, Cinara Vasconcelos da et al. Alkaloids and other metabolites from stems and fruits of Zanthoxylum tingoassuiba A. St. Hil. Química Nova, v. 31, n. 8, p. 2071-2075, 2008.

SILVA, Jordana Benfica et al. Estudo de identificação de metabólitos secundários no caule e na raiz do milho (Zea mays). In: Congresso Mineiro de Engenharias e ArquiteturaCENAR. 2017.

SILVA, P. B. et al. Avaliação do potencial alelopático, atividade antimicrobiana e antioxidante dos extratos orgânicos das folhas de Pyrostegia venusta (KerGawl.) Miers (Bignoniaceae). Revista Brasileira de Plantas Medicinais, p. 447-455, 2011. 